\title{
Quantum Phase Transitions
}

\author{
M. Lavagna* \\ Commissariat à l'Energie Atomique, DRFMC /SPSMS, \\ 17, rue des Martyrs, \\ 38054 Grenoble Cedex 9, France
}

\begin{abstract}
We give a general introduction to quantum phase transitions in strongly-correlated electron systems. These transitions which occur at zero temperature when a non-thermal parameter $g$ like pressure, chemical composition or magnetic field is tuned to a critical value are characterized by a dynamic exponent $z$ related to the energy and length scales $\Delta$ and $\xi$. We show how one can derive an effective bosonic model associated with the fluctuations of the ordering fields. Simple arguments based on an expansion to first order in the effective interaction $u$ allow to define an upper-critical dimension $D_{C}=4$ (where $D=d+z$ and $d$ is the spatial dimension) below which mean-field description is no longer valid. We present an alternative tricritical crossover approach valid at $D<D_{C}$ in the large- $N$ limit. We emphasize the role of pertubative renormalization group (RG) approaches and self-consistent renormalized spin fluctuation (SCR-SF) theories to understand the quantum-classical crossover in the vicinity of the quantum critical point with generalization to the Kondo effect in heavy-fermion systems. Finally, we quote some recent inelastic neutron scattering experiments performed on heavy-fermions which lead to unusual scaling law in $\omega / T$ for the dynamical spin susceptibility revealing critical local modes beyond the itinerant magnetism picture.We mention new attempts to describe this local quantum critical point.
\end{abstract}

\section{Introduction}

The recent years have seen a renewal of interest in the study of quantum phase transitions (QPT) motivated by experiments in cuprate superconductors, heavy fermion compounds, organic conductors and related systems (Santa Barbara Proceedings 1996, Sachdev 1999). By definition a quantum phase transition is a phase transition which occurs at zero temperature when a non-thermal parameter $g$ like pressure, chemical composition or magnetic field is tuned to a critical value. The transition is characterized by quantum fluctuations even in the ground state due to Heisenberg's uncertainty principle. It is usually accompanied by a drastic change in the behavior of the correlation functions.

In these lectures, we will focus on the quantum phase transitions of second order. The associated fluctuations are characterized by two different quantities that are respectively the energy scale $\Delta$ and the correlation length $\xi$. In the case when the energy spectrum has a gap, $\Delta$ measures the difference of energy between the ground state and the lowest excited level. If the spectrum is gapless, one can still define $\Delta$ as a pseudo-gap, splitting the lower-energy and the higher-energy parts in the density fluctuation spectrum. Generally speaking $\Delta$ vanishes at the transition which occurs at a critical value $g_{c}$ of a dimensionless parameter $g$

$$
\Delta \sim\left|g-g_{c}\right|^{z \nu}
$$

where $z \nu$ is a critical exponent which takes a universal value independent of the microscopic details of the hamiltonian describing the system. One will denote by $\Delta_{+}$and $\Delta_{-}$the values of the gap respectively above and below $g_{c}$. Apart from an eventual constant of proportionality, both quantities are characterized by the same critical exponent $z \nu$.

The other scale is provided by the correlation length $\xi$. It is defined as the length scale characterizing the exponential decay of the equal-time correlation function $\langle M(\mathbf{r}, t) M(\mathbf{0}, t)\rangle$ in the disordered phase at $T=0$. The length scale $\xi$ diverges at the quantum critical point $(\mathrm{QCP})$ defined at $T=0$ and $g=g_{c}$ according to

$$
\xi^{-1} \sim\left|g-g_{c}\right|^{\nu}
$$

Putting together the vanishing of $\Delta$ and the diverging of $\xi$ at the transition, one can write

$$
\Delta \sim \xi^{-z}
$$

where $\mathrm{z}$ is the dynamic exponent. Those critical exponents are all defined at $T=0$ and $\mathrm{g} \rightarrow g_{c}$. However, they are also relevant at finite temperature and $g \neq g_{c}$. 
Figure 1 displays the schematic phase diagram $(T, g)$ that can be derived. The full line is the line of phase transitions separating the long-range ordered and the disordered phases. The dotted line corresponds to the Ginzburg temperature $T_{G}$ below which classical fluctuations are important. In the classical critical region, an effective classical theory applies. The regime $I$ is the quantum disordered regime driven by quantum fluctuations as resulting from the Heisenberg's uncertainty principle. Oppositely only thermal fluctuations are relevant in the regime $I I I$ above $T_{G}$. Finally, the regime $I I$ is the quantum critical regime characterized by a rich interplay of effects driven by both quantum and thermal fluctuations.

\section{Experimental examples}

We will mention some recent experimental studies of second-order quantum phase transitions.

(i) $\mathrm{LiHoF}_{4}$

In the insulator $\mathrm{LiHoF}_{4}$, the $\mathrm{Ho}$ ions fluctuate between two spin states depending whether the spins are parallel or antiparallel to a particular crystalline axis. At zero temperature, applying an external magnetic field perpendicular to the magnetic axis makes the system go from a ferromagnetic ground state to a quantum paramagnetic state in which long-range ferromagnetic order is destroyed due to quantum tunneling between the two spin states (Bitko et al, 1996). The quantum critical point is reached at a critical value $H_{C}$ of the magnetic field. Raising the temperature also destroys the long-range magnetic order driving the system to a high-temperature magnetically disordered state. Note that the latter state, the thermal paramagnet is fundamentally different in nature from the quantum paramagnet formed at zero temperature.

(ii) Heavy fermion compounds

One of the most striking properties of heavy fermion compounds discovered these last years is the existence of a quantum phase transition driven by chemical composition change, pressure or magnetic field. For example, $C e C u_{6-x} A u_{x}$ (von Lohneysen et al, 1994) and $C e_{1-x} L a_{x} R u_{2} S i_{2}$ (Raymond et al, 1997) present a transition from a magnetically disordered phase to a long-range antiferromagnetic order respectively at $x_{C}=0.1$ and $x_{C}=0.08$. We will mention other sytems as $\mathrm{CeIn}_{3}, \mathrm{CePd}_{2} \mathrm{Si}_{2}$ (Mathur et al, 1998), $\mathrm{CeNi} \mathrm{Ge}_{2}$ (Steglich, 1996) and $U_{1-x} Y_{x} P d_{3}$ (Seaman et al, 1991) exhibiting the same behavior. Once a long-range magnetic order is set up, the effect of a pressure or a magnetic field is to drive the system back to a magnetically disordered phase. Remarkably while far from the quantum critical point, the magnetically disordered phase is a Fermi liquid with large effective masses associated with the formation of heavy quasiparticles, there are some indications that the thermal properties of the system in the disordered phase close to the quantum critical point differ from that of a Fermi liquid (Santa Barbara Proceedings, 1996 and Coleman, 1999). Typically in $C e C u_{5.9} A u_{0.1}$ (von Lohneysen et al, 1994), the specific heat $C$ depends on $T$ as $C / T \sim-\ln \left(T / T_{0}\right)$, the magnetic susceptibility as $\chi \sim 1-\alpha \sqrt{T}$, and the $T$-dependent part of the resistivity as $\Delta \rho \sim T$ instead of $C / T \sim \chi \sim$ Const and $\Delta \rho \sim T^{2}$ in the Fermi liquid state. The origin of this non-Fermi liquid (NFL) behavior is presently a problem of considerable debate.

An important element in the knowledge of the quantum critical point has been recently brought by inelastic neutron scattering (INS) experiments performed on a $C e C u_{5.9} A u_{0.1}$ single-crystal. The dynamical spin susceptibility $\chi "(\mathbf{q}, \omega)$ near the magnetic instability wavevector $\mathbf{Q}$ has been found to obey an anomalous $\omega / T$ scaling law as a function of temperature

$$
\chi "(\mathbf{Q}, \omega) \sim T^{-\alpha} g(\omega / T)
$$

with $\alpha$ of order 0.75 . Moreover, such a $\omega$ and $T$ dependence stands over the entire Brillouin zone revealing in the bulk susceptibility too. This fact strongly suggests that the spin dynamics are critical not only at large length scales but also at atomic length scales contrary to what happens in the traditional itinerant magnetism picture. We will come back to this point at the end of the paper.

Let us focus now on the general problem of quantum phase transitions between a Fermi liquid and a magnetically ordered phase. This is still a largely controversial topic in which many aspects are not fully understood. Details of the topology of the Fermi surface often matters introducing important nesting effects and Kohn anomalies in the spin susceptibility when the magnetic wavevector spans the Fermi surface. For reasons of simplicity, we will not consider those effects of nesting. 
The rest of the paper is organized as follows. In section 3, we show how one can integrate out the fermion fields to derive an effective bosonic model associated with the fluctuations of the ordering fields. The resulting effective action exhibits a dynamic exponent $z$ equal to 3 in the vicinity of a ferromagnetic critical point and 2 for the antiferromagnetic case. In section 4 , we give simple arguments based on an expansion to first order in the effective interaction $u$ to determine an upper-critical dimension $D_{C}=4$ (where $D=d+z$ and $d$ is the spatial dimension) below which first order fluctuations diverge and mean-field description is no longer valid. Section 5 exposes an alternative tricritical crossover approach valid at $D<D_{C}$ in the large- $N$ limit. Section 5 gives a short presentation of pertubative renormalization group (RG) approaches and their links to self-consistent renormalized spin fluctuation (SCR-SF) theories to understand the quantum-classical crossover in the vicinity of the quantum critical point. Section 6 generalizes the approach to the Kondo lattice model which is believed to describe the heavy-fermion situation Finally, we quote some recent inelastic neutron scattering experiments performed on heavy-fermions leading to unusual scaling law in $\omega / T$ for the dynamical spin susceptibility which reveals critical local modes beyond the itinerant magnetism picture inherent to pertubative RG and SCR-SF approaches. We mention new attempts to describe this local quantum critical point.

\section{Derivation of an effective bosonic theory}

We start from the Hubbard hamiltonian which represents the archetypal model for correlated electron system. For a single nondegenerate band of electrons of spin $1 / 2$, the hamiltonian is

$$
H=\sum_{k \sigma} \varepsilon_{k} c_{k \sigma}^{\dagger} c_{k \sigma}+U \sum_{i} n_{i \uparrow} n_{i \downarrow}
$$

where the last term the Coulomb repulsion term expressed in Wannier representation, $\varepsilon_{k}=-\left[\sum_{\delta} t_{i j} \exp (i \mathbf{k} . \delta)\right] / z, \mathrm{t}_{i j}$ is the overlap integrals between neighboring sites and $z$ the coordination number. The hamiltonian is characterized by a competition between the kinetic term and the Coulomb interaction $U$ which generally induces a quantum phase transition from a Fermi liquid to a magnetically ordered state at a critical value of $U / t$.

By integrating out the fermion fields, we will first show how from the Hubbard hamiltonian, we can derive an effective bosonic theory in terms of the fluctuations of the ordering fields $\Phi_{i}$ associated with the magnetizations. Following (Hertz and Klenin, 1974), we decompose the proof into the following steps. The calculations are performed in the magnetically-disordered phase.

First let us write the Coulomb term as a function of charge and spin density variables

$$
U \sum_{i} n_{i \uparrow} n_{i \downarrow}=\frac{U}{4} \sum_{i}\left(n_{i \uparrow}+n_{i \downarrow}\right)^{2}-\frac{U}{4} \sum_{i}\left(n_{i \uparrow}-n_{i \downarrow}\right)^{2}
$$

where $\left(n_{i \uparrow}-n_{i \downarrow}\right) / 2=\Phi_{i}^{z}$ is the $z$-magnetization. Note that one can extend the writing to all three directions $x, y$ and $z$ in order to preserve the spin rotation invariance.

Let us consider the functional integral of the partition function

$$
\begin{array}{r}
Z=\int \mathcal{D} c_{i \sigma} \exp \left[-\int_{0}^{\beta} \mathcal{L}(\tau) d \tau\right] \\
\mathcal{L}(\tau)=\sum_{i \sigma} c_{i \sigma}^{\dagger} \partial_{\tau} c_{i \sigma}+H(\tau)
\end{array}
$$

and perform a Hubbard-Stratonovich transformation on the Coulomb interaction term. Using the identity

$$
\int d \Phi_{i}^{z}(\tau) \exp \left[-\int_{0}^{\beta}\left(\Phi_{i}^{z}(\tau)-\sqrt{U} c_{i \sigma_{1}}^{\dagger} \tau_{\sigma_{1} \sigma_{2}}^{z} c_{i \sigma_{2}}\right)\left(\Phi_{i}^{z}(\tau)-\sqrt{U} c_{i \sigma_{3}}^{\dagger} \tau_{\sigma_{3} \sigma_{4}}^{z} c_{i \sigma_{4}}\right) d \tau\right]=1
$$

in which the summation on the spin indices $\sigma_{i}$ are implicit and $\tau^{i}$ represent the Pauli matrices, we find

$$
Z=Z_{0} \int \mathcal{D} c_{i \sigma} d \Phi_{i}^{z}(\tau) \exp \left[-\int_{0}^{\beta}\left(\Phi_{i}^{z}(\tau) \Phi_{i}^{z}(\tau)-\sqrt{U} \Phi_{i}^{z}(\tau) c_{i \sigma}^{\dagger} \tau_{\sigma \sigma^{\prime}}^{z} c_{i \sigma^{\prime}}\right) d \tau\right]
$$


Integrating out the grassmannian variables $c_{i \sigma}$, we get

$$
\begin{gathered}
Z=Z_{0} \int d \Phi_{i}^{z}(\tau) \exp \left[-S_{\text {eff }}\left(\Phi_{i}\right)\right] \\
S_{\text {eff }}\left(\Phi_{i}\right)=\int_{0}^{\beta} \int_{0}^{\beta}\left[\Phi_{i}^{z}(\tau) \Phi_{i}^{z}(\tau) \delta\left(\tau-\tau^{\prime}\right)-\operatorname{Tr} \ln \left(1-\sqrt{U} \Phi_{i}(\tau) G_{0}^{i j}\left(\tau-\tau^{\prime}\right)\right)\right] d \tau d \tau^{\prime}
\end{gathered}
$$

where $G_{0}$ is the bare Green function of electrons at $U=0$.

Expanding the Trln term up to the second and fourth order in $\Phi$, we have

$$
\begin{aligned}
S_{e f f}\left(\Phi_{i}\right)= & \beta V \sum_{q, i \omega_{\nu}}\left(1-U \chi_{0}\left(q, i \omega_{\nu}\right)\right) \Phi\left(q, i \omega_{\nu}\right) \Phi\left(-q,-i \omega_{\nu}\right) \\
& +u \beta V^{4} \sum_{q_{i}, i \omega_{i}} \Phi\left(q_{1}, i \omega_{1}\right) \Phi\left(q_{2}, i \omega_{2}\right) \Phi\left(q_{3}, i \omega_{3}\right) \Phi\left(-q_{1}-q_{2}-q_{3},-i \omega_{1}-i \omega_{2}-i \omega_{3}\right)
\end{aligned}
$$

where $\chi_{0}\left(q, i \omega_{\nu}\right)$ is the bare dynamical susceptibility and $u$ an effective interaction assumed to be local.

Close to a ferromagnetic phase transition, we can use the Lindhard expansion of $\chi_{0}(q, \omega)$ around $q=\omega=0$

$$
\chi_{0}\left(q, i \omega_{\nu}\right)=\chi_{0}(0,0)-b \frac{q^{2}}{k_{F}^{2}}+i a \frac{\omega}{\Gamma_{q}}
$$

where $k_{F}$ is the Fermi wavevector, $a$ and $b$ are constants and $\Gamma_{q}=q v_{F}$ is the relaxation rate which vanishes in the $q \rightarrow 0$ limit. Note that this vanishing is imposed by some symmetry arguments due to the fact that the fluctuations of the order parameter are conserved in the ferromagnetic case. Hence one can draw the effective bosonic action in the ferromagnetic case

$$
\begin{aligned}
S_{e f f}\left(\Phi_{i}\right)= & \beta V \sum_{q, i \omega_{\nu}}\left(\delta+q^{2}+\left|\omega_{\nu}\right| / q\right) \Phi\left(q, i \omega_{\nu}\right) \Phi\left(-q,-i \omega_{\nu}\right) \\
& +u \beta V^{4} \sum_{q_{i}, i \omega_{i}} \Phi\left(q_{1}, i \omega_{1}\right) \Phi\left(q_{2}, i \omega_{2}\right) \Phi\left(q_{3}, i \omega_{3}\right) \Phi\left(-q_{1}-q_{2}-q_{3},-i \omega_{1}-i \omega_{2}-i \omega_{3}\right)
\end{aligned}
$$

where $\delta=1-U \chi_{0}\left(q, i \omega_{\nu}\right)$ called the Stoner factor measures the distance to the magnetic instability.

The result can be generalized to the case of an antiferromagnetic instability. In this case if we denote by $q$ the deviation to the antiferromagnetic wavevector $Q=(\pi, \pi, \pi)$, the expansion expressed in Eq. (12) is still valid around $Q$. However since the fluctuations are not conserved in the antiferromagnetic case, the relaxation rate $\Gamma_{q}$ is now $q$-independent. The first term of Eq (13) is modified in the following way

$$
S_{e f f}^{(2)}\left(\Phi_{i}\right)=\beta V \sum_{q, i \omega_{\nu}}\left(\delta+q^{2}+\left|\omega_{\nu}\right|\right) \Phi\left(q, i \omega_{\nu}\right) \Phi\left(-q,-i \omega_{\nu}\right)
$$

The justification of the expansion of the Trln term in Eq. (10) up to the second and fourth order in $\Phi$ lies in the fact that $\Phi_{i}$ representing the fluctuations of the magnetization are expected to be small in the magnetically-disordered phase close to the quantum critical point. However, the expansion would not stand within the long-range ordered state where the determination of the finite magnetization $M_{0}$ which $\Phi$ can be expanded about $\left(\Phi=M_{0}+\delta \Phi\right)$ requires to minimize the full expression of the free energy including all orders and not stop to the fourth order. This is precisely what the equivalent of the gap equation for the magnetization does.

\section{Pertubation theory in $u$ : existence of an upper-critical dimension}

In the effective bosonic theory presented in the previous section, $q$ and $\omega$ do not appear at the same order. They may do so in some different models. For convenience in this section, we will only consider the case when $\omega$ appears at the 2nd order too and use the relastivistic notation $Q^{2}=q^{2}+\omega^{2}$. We will show the upper-critical dimension to be equal to 4 for $D=d+1$ as a result of diverging critical fluctuations when $D<4$. The result will be generalized later on when a dynamic exponent $z$ instead of 1 makes $D$ change to $d+z$ keeping the same value of 4 for the upper-critical dimension. In the realistic notations, the effective action writes 


$$
\begin{aligned}
S_{e f f}\left(\Phi_{i}\right)= & \beta V \sum_{q, i \omega_{\nu}}\left(\delta+Q^{2}\right) \Phi(Q) \Phi(-Q) \\
& +u \beta V^{4} \sum_{q_{i}, i \omega_{i}} \Phi\left(Q_{1}\right) \Phi\left(Q_{2}\right) \Phi\left(Q_{3}\right) \Phi\left(-Q_{1}-Q_{2}-Q_{3}\right)
\end{aligned}
$$

At zero temperature and to zeroth order in $u$, the spin susceptibility $\chi(Q)$ related to the correlation function in $\Phi$ is given by

$$
\left[\chi^{(0)}(Q)\right]^{-1}=Q^{2}+\delta
$$

The uniform static susceptibility $\chi^{(0)}(0)$ diverges at $\delta_{c}^{(0)}=0 . \quad \delta$ plays the role of a tuning parameter and the quantum phase transition takes place at $\delta_{c}^{(0)}=0$.

To first order in $\mathrm{u}$, the result is changed in the following way

$$
[\chi(Q)]^{-1}=Q^{2}+\delta+\frac{u}{2} \int \frac{d^{D} Q}{(2 \pi)^{D}} \frac{1}{Q^{2}+\delta}
$$

where $D=d+1$ and $d$ is the spatial dimension. The critical value of $\delta$ is given by

$$
\delta_{c}=-\frac{u}{2} \int \frac{d^{D} Q}{(2 \pi)^{D}} \frac{1}{Q^{2}+\delta_{c}}
$$

Denoting $s=\delta-\delta_{c}$ which measures the deviation of the system from the QCP

$$
[\chi(Q)]^{-1}=Q^{2}+s+\frac{u}{2} \int \frac{d^{D} Q}{(2 \pi)^{D}}\left[\frac{1}{Q^{2}+\delta}-\frac{1}{Q^{2}}\right]
$$

For $D>4$, the integrand diverges in the $Q \rightarrow \infty$ limit and one needs to introduce a cut-off $\Lambda$ for $Q$. One can then perform an expansion in $\mathrm{s}$

$$
[\chi(Q)]^{-1}=Q^{2}+s\left[1-c_{1} u \Lambda^{D-4}\right]
$$

where $c_{1}$ is a nonuniversal constant depending on the nature of the cut-off. $\chi(0)$ diverges in the $s \rightarrow 0$ limit. Mean-field critical properties still apply with small first order corrections.

For $D<4$, the integrand now converges in the $Q \rightarrow \infty$ limit. Therefore under the condition $u \ll \Lambda^{D-4}$, one can put $\Lambda \rightarrow \infty$ in the integral. To first order in $u$

$$
[\chi(Q)]^{-1}=Q^{2}+s\left[1-\frac{1}{2} \frac{2 \Gamma\left(\frac{4-D}{2}\right)}{(D-2)(4 \pi)^{D / 2}} \frac{u}{s^{(4-D) / 2}}\right]
$$

As small as $u$ is, the correction to the mean-field result is important. It even diverges in the $s \rightarrow 0$ limit. One can then deduce an upper-critical value for the dimension $D_{C}=4$. At $D<D_{C}$ mean-field results are not correct and a more sophisticated resummation of the pertubation expansion is required. For this purpose, we now present a large $\mathrm{N}$ theory introducing a tricritical crossover function which allows to do that.

\section{Large- $N$ theory and tricritical crossovers}

For $D<4$, Eq. (21) established at first order in $u$ suggests that $[\chi(Q)]^{-1}$ can be expressed as

$$
[\chi(Q)]^{-1}=s \psi_{D}(q, v)
$$

where we have denoted by $q=Q / s^{1 / 2}$ and $v=u / s^{(4-D) / 2}$ and introduced $\psi_{D}[q, v]$ as a universal function called the tricritical crossover function (Brézin and Zinn-Justin, 1985 and Sachdev, 1999). To first order in $u, \psi_{D}(q, v)$ can be identified with

$$
\psi_{D}(q, v)=q^{2}+1-\frac{\Gamma\left(\frac{4-D}{2}\right)}{(D-2)(4 \pi)^{D / 2}} v+\mathcal{O}\left(v^{2}\right)
$$


If we assume that we can put $\Lambda \rightarrow \infty$ in all the higher-order terms in $u$, we can expect $[\chi(Q)]^{-1}$ to take the form expressed in Eq. 222).

We now explain how in the large $N$ limit $\psi_{D}(q, v)$ can be determined at any order in $v$. To do that, we first extend the previous descriptions to any value of the degeneracy of the Hubbard-Stratonovich parameter $\Phi_{\alpha}, \alpha=1,2 \ldots N$ and let $N$ go to $\infty$ at the end of the calculation. Performing a Hubbard-Stratonovich transformation on the the $\Phi^{4}$-term, one gets in the large-N limit

$$
[\chi(Q)]^{-1}=Q^{2}+\delta+u \frac{N+2}{6}\left\langle\Phi^{2}\right\rangle
$$

where $\left\langle\Phi^{2}\right\rangle$ is self-consistently determined as in the self-consistent one-loop approximation according to

$$
\left\langle\Phi^{2}\right\rangle=\int \frac{d^{D} Q}{(2 \pi)^{D}} \frac{1}{Q^{2}+\delta+u \frac{N+2}{6}\left\langle\Phi^{2}\right\rangle}
$$

Hence $\delta_{C}$ is defined by

$$
\delta_{C}+\left.u \frac{N+2}{6}\left\langle\Phi^{2}\right\rangle\right|_{\delta=\delta_{C}}=0
$$

and

$$
[\chi(Q)]^{-1}=Q^{2}+\left(\delta-\delta_{C}\right)+u \frac{N+2}{6}\left[\left\langle\Phi^{2}\right\rangle-\left.\left\langle\Phi^{2}\right\rangle\right|_{\delta=\delta_{C}}\right]
$$

The critical crossover function $\psi_{D}(q, v)$ can be identified with

$$
\psi_{D}(q, v)=q^{2}+\pi_{D}(v)
$$

where the function $\pi_{D}(v)$ is solution of the following nonlinear equation

$$
\pi_{D}(v)+N v \frac{\Gamma\left(\frac{4-D}{2}\right)}{3(D-2)(4 \pi)^{D / 2}}\left[\pi_{D}(v)\right]^{(D-2) / 2}=1
$$

In the $v \rightarrow \infty$ limit, $\pi_{D}(v) \sim v^{-2 /(D-2)}$ and $[\chi(0)]^{-1}$ behaves as $s^{D /(D-2)}$ at small $s$. The latter result on $[\chi(0)]^{-1}$ settles the difficulties arising from the diverging correction term obtained to first order in u when $D<D_{C}$.

\section{Pertubative renormalization group}

We send the reader to a number of very good reviews existing in the litterature on this sophistical and powerful approach. We will simply try to clear out the essential points involved in the method. Let us start again from the effective action derived in section 4

$$
\begin{aligned}
S_{e f f}(\Phi)= & \frac{1}{2} \sum_{q, \omega}\left(\delta+q^{2}+|\omega| / q\right)|\Phi(q, \omega)|^{2} \\
& +\frac{1}{2} u \frac{1}{\beta \mathcal{N}} \sum_{q_{i}, \omega_{i}} \Phi\left(q_{1}, \omega_{1}\right) \Phi\left(q_{2}, \omega_{2}\right) \Phi\left(q_{3}, \omega_{3}\right) \Phi\left(-q_{1}-q_{2}-q_{3},-\omega_{1}-\omega_{2}-\omega_{3}\right)
\end{aligned}
$$

where $\mathcal{N}$ is the number of sites and $\beta$ is the inverse temperature. The general idea of the renormalization group approach is to get rid of the short-range and short-time details of the fluctuations of the order parameter to derive a renormalized effective action in which the different parameters are rescaled. As shown in Figure 2 the aim is then to eliminate out the contribution to $\Phi(q, \omega)$ from the outer-shell with large values of $q$ and $\omega$. We also define the complementary inner-shell characterized by small values of $q$ and $\omega$. Following Hertz' paper (Hertz, 1976), we show how to derive the scaling equations along two steps : first keeping the inner-shell contribution only, and then adding the remaining contribution of the outer-shell. This provides us with two scaling equations for $\delta$ and $u$, respectively the 
Stoner factor and the effective interaction. We will mention how later on, Millis showed how it is crucial to consider an additional scaling equation for temperature.

( $i$ ) eliminating out the outer-shell within the inner shell contribution to $S_{\text {eff }}$.

First, let us remove the outer-shell contribution to $\Phi(q, \omega)$. Since $q$ and $\omega$ appears in the quadratic term of $S_{e f f}(\Phi)$ at different orders, an anisotropic scaling procedure is required. The outer-shell is defined by

$$
\begin{array}{r}
\exp (-l)<q<1 \\
\exp (-z l)<\omega<1
\end{array}
$$

where $l$ is infinitesimal and $z$ will be proved later on to coincide with the dynamic exponent. Retaining the contribution to $S_{\text {eff }}(\Phi)$ of the inner-shell only with small $q$ and $\omega$ values, one gets to the second order

$$
S_{\text {eff }}^{(2)}(\Phi)=\frac{1}{2} \beta \mathcal{N} \int_{0}^{\exp (-l)} \frac{d^{d} q}{(2 \pi)^{d}} \int_{0}^{\exp (-z l)} \frac{d \omega}{2 \pi}\left(\delta^{\prime}+q^{2}+|\omega| / q\right)|\Phi(q, \omega)|^{2}
$$

The change from $\delta$ to $\delta^{\prime}$ is of order $l$.

Next step consists in rescaling the variables $q$ and $\omega$ in the following way

$$
\begin{aligned}
q^{\prime} & =q \exp (l) \\
\omega^{\prime} & =\omega \exp (z l)
\end{aligned}
$$

With this change of variables, we have

$$
\begin{aligned}
S_{\text {eff }}^{(2)}(\Phi)= & \frac{1}{2} \beta \mathcal{N} \exp [-(d+z) l] \int_{0}^{1} \frac{d^{d} q^{\prime}}{(2 \pi)^{d}} \int_{0}^{1} \frac{d \omega^{\prime}}{2 \pi}\left(\delta^{\prime}+q^{\prime 2} \exp (-2 l)+\left|\omega^{\prime}\right| / q^{\prime} \exp [-(z-1) l]\right) \\
& \left|\Phi\left(q^{\prime} \exp (-l), \omega^{\prime} \exp (-z l)\right)\right|^{2}
\end{aligned}
$$

The fields $\Phi(q, \omega)$ are then rescaled so that the terms in $q^{\prime 2}$ and $\left|\omega^{\prime}\right| / q^{\prime}$ in $S_{e f f}^{(2)}(\Phi)$ are unchanged. Thanks to the anisotropic scaling procedure introduced earlier, we are allowed to do that as soon as we choose $z$ equal to the dynamic exponent 3 in the ferromagnetic case. Introducing the scaling

$$
\Phi^{\prime}\left(q^{\prime}, \omega^{\prime}\right)=\Phi(q, \omega) \exp [-(d+z+2) l / 2]
$$

one gets

$$
S_{e f f}^{(2)}(\Phi)=\frac{1}{2} \beta \mathcal{N} \int_{0}^{1} \frac{d^{d} q^{\prime}}{(2 \pi)^{d}} \int_{0}^{1} \frac{d \omega^{\prime}}{2 \pi}\left(\exp (2 l) \delta^{\prime}+q^{\prime 2}+\left|\omega^{\prime}\right| / q^{\prime}\right)\left|\Phi\left(q^{\prime}, \omega^{\prime}\right)\right|^{2}
$$

We can see from this expression that the initial form of $S_{\text {eff }}^{(2)}(\Phi)$ is recovered provided that $\exp (2 l) \delta^{\prime}=\delta$. This gives us the first part of the scaling equation for $\delta$

$$
\frac{d \delta}{d l}=2 \delta
$$

In the same way, the effective action to the 4 th order in $u$ can be expressed as

$$
\begin{aligned}
S_{\text {eff }}^{(4)}(\Phi)= & \frac{1}{4}(\beta \mathcal{N})^{2} u^{\prime} \exp [-3(d+z) l] \exp [4(d+z+2) l / 2] \int_{0}^{1} \frac{d^{d} q_{i}^{\prime}}{(2 \pi)^{d}} \int_{0}^{1} \frac{d \omega_{i}^{\prime}}{2 \pi} \\
& \Phi\left(q_{1}, \omega_{1}\right) \Phi\left(q_{2}, \omega_{2}\right) \Phi\left(q_{3}, \omega_{3}\right) \Phi\left(-q_{1}-q_{2}-q_{3},-\omega_{1}-\omega_{2}-\omega_{3}\right)
\end{aligned}
$$

where the summation $\prod_{i=1,2,3}$ on the subscript $i$ is implicit. In order to keep the quartic term unchanged, one needs to transform $u$ according to

$$
u^{\prime} \exp (\varepsilon l)=u
$$

with $\varepsilon=4-(d+z)$ enabling us to write the second scaling equation for $u$

$$
\frac{d u}{d l}=\varepsilon u
$$


As for $\delta$, we will show in (ii) that the latter scaling equation should be laced with a second term in the righthand side to be complete. Before ending up with $(i)$, let us point out that the scaling equation (40) provides an alternative way to define the upper-critical dimension for $D=d+z$ generalizing to any $z$ the result previously obtained in section 4. The spatial dimension is increased by the dynamic exponent $z$ equal to 3 in the ferromagnetic case and to 2 in the antiferromagnetic case. Above the upper-critical dimension found to be $D_{C}=4, \varepsilon$ has a negative sign and $u$ is rescaled to zero. We are then left with the quadratic term of $S_{\text {eff }}$ only and the system reaches a gaussian fixed point. Oppositely below $D_{C}=4, \varepsilon$ has a positive sign. The interaction is relevant and a non-gaussian fixed point is reached.

(ii) incorporating the remaining contribution of the outer-shell to $S_{\text {eff }}$.

The last step consists in considering the missing contribution with large values of $q$ and $\omega$. If one denotes by $\sum_{q, \omega}^{\prime}$ and $\sum_{q, \omega}^{\prime \prime}$ respectively the summation over the inner- and the outer-shells, the corresponding correction to $S_{\text {eff }}^{(4)}(\Phi)$ is

$$
\begin{aligned}
S_{\text {eff }}^{(4)}(\Phi)= & \frac{u}{4 \beta \mathcal{N}} \sum_{q, \omega} \prod_{i=1}^{4} \Phi\left(q_{i}, \omega_{i}\right) \delta\left(\sum_{i=1}^{4} q_{i}\right) \delta\left(\sum_{i=1}^{4} \omega_{i}\right) \\
& +\frac{3 u}{2 \beta \mathcal{N}} \sum_{q, \omega} "|\Phi(q, \omega)|^{2} \sum_{q, \omega}{ }^{\prime}|\Phi(q, \omega)|^{2}
\end{aligned}
$$

The two terms in the righthand side of the last equation corresponds to the interaction of one particle in the outer-shell with one particle respectively in the outer-shell and in the inner one. Part of the action which is quadratic as $\sum_{q, \omega}{ }^{\prime}|\Phi(q, \omega)|^{2}$ can be resummed. The final expression for $S_{\text {eff }}$ including all contributions from the inner- and the outer-shell up to the 4 th order is

$$
\begin{aligned}
S_{\text {eff }}^{(2)}(\Phi)= & \frac{1}{2} \sum_{q, \omega} "\left(\delta+q^{2}+|\omega| / q\right)|\Phi(q, \omega)|^{2} \\
& +\frac{u}{4 \beta \mathcal{N}} \sum_{q, \omega} " \prod_{i=1}^{4} \Phi\left(q_{i}, \omega_{i}\right) \delta\left(\sum_{i=1}^{4} q_{i}\right) \delta\left(\sum_{i=1}^{4} \omega_{i}\right) \\
& +\frac{1}{2} \sum_{q, \omega}{ }^{\prime} \ln \left[\delta+q^{2}+|\omega| / q+\frac{3 u}{\beta N} \sum_{q, \omega} "|\Phi(q, \omega)|^{2}\right]
\end{aligned}
$$

Expanding the $l n$ term up to the 4 th order in $\Phi(q, \omega)$ enables us to get the additional part of the scaling equations. Putting it together with the truncated part of the scaling equations obtained in step $(i)$, one can write the complete scaling equations for $\delta$ and $u$

$$
\begin{aligned}
& \frac{d \delta}{d l}=2 \delta+\frac{3 u}{\beta \mathcal{N}} \sum_{q, \omega}{ }^{\prime}\left(\delta+q^{2}+|\omega| / q\right)^{-1} \\
& \frac{d u}{d l}=\varepsilon u-\frac{9 u^{2}}{\beta \mathcal{N}} \sum_{q, \omega}{ }^{\prime}\left(\delta+q^{2}+|\omega| / q\right)^{-2}
\end{aligned}
$$

These two equations constitute the whole set of renormalization group equations derived by Hertz. The crossover temperature $T_{I}$ separating the quantum to the classical regime is then defined in the following way. At the end of the scaling procedure, the quantum regime is reached if only the $\nu=0$ term contributes in the Matsubara frequency sum of Eqs. (43, 44). Later on, Millis (Millis, 1993) corrected Hertz' paper and showed that the temperature should be rescaled as well as $\delta$ and $u$ adding a third renormalization group equation for temperature

$$
\frac{d T}{d l}=z T
$$

Then the criterion used to differenciate the quantum from the classical regime is slightly different. It depends on how the temperature $T^{*}$ at the end of the scaling procedure compares to an energy cut-off $\omega^{*}$.

We will now give a physical interpretation to $T_{I}$ in terms of the spectrum of spin excitations as schematized in Figure 3 (). The shaded region corresponds to the Lindhard continuum of electron-hole pair excitations. It is defined by a finite value of the imaginary part of the bare dynamical susceptibility. The collective mode obtained from the poles of the inverse renormalized susceptibility is a damped magnon as reported in Figure 3. In the ferromagnetic case, 
the dispersion of the collective mode changes from a $q$ to a $q^{3}$ dependence at a characteristic value of the wavevector $q^{*}=\xi^{-1} \sim \sqrt{\delta}$. The energy-scale $\omega^{*}$ corresponds to the energy of the collective mode on the scale of the magnetic length $\xi$. The regime is either quantum or classical depending whether $T^{*}$ is found to be smaller or larger than $\omega^{*}$. The two regimes are characterized by different temperature behaviors of the physical quantities as for instance the magnetic correlation length $\xi$ and the coefficient $\gamma$ of the linear temperature dependence of the specific heat. Above a second crossover temperature $T_{I I}$, all the relevant modes contributing to Eqs. (43) and (44) have energies much less that $k_{B} T$ and the physical quantities are driven by the temperature only. The results obtained by Millis are similar to those reported in Figure 4 and only depend on the spatial dimension $d$ and on the dynamic exponent $z$.

Note that an alternative approach to the magnetic phase transitions in the Hubbard model has been developed in (Moriya and Kawabata, 1973) based on a self-consistent renormalized theory of spin fluctuations (SCR-SF). It leads to results very similar to those obtained by the pertubative renormalization group. Comparisons between the two approaches can be found in (Millis, 1993).

\section{Heavy-Fermions and the Kondo lattice model}

The model that is believed to describe the physics of heavy fermions is the Kondo lattice model where impurity spins distributed on the sites of a periodic lattice interact with the spin of local conduction electrons via a Kondo coupling $J_{K}$. The main features of the model result from the competition between two energy scales (Doniach, 1977): the Kondo temperature $T_{K}$ corresponding to the binding energy at zero temperature of the singlet formed by the impurity spin screened by the spin of the conduction electrons; and the Ruderman-Kittel Kasuya-Yosida -RKKYinteractions among neighboring spins mediated by the conduction electrons. The latter one favors the formation of a long-range magnetic order while the former one blocks it by Kondo screening. The consequence is the existence of a quantum critical point at a critical value of $J_{K}$ below which a long-range magnetic order occurs. In the case when the total number of conduction electrons is less than one per site, all phases are in a metallic state.

The large $N$ expansions (Millis, 1987) which have been carried out for these models (where $N$ simultaneously represents the degeneracy of the conduction electrons and of the spin channels) are known to give a good description of the Kondo effect but fail to account for the spin-fluctuations since the RKKY interactions only appear at the order $1 / N^{2}$. With the aim to describe the critical phenomena around the quantum critical point of the heavy-fermion systems, it has been recently proposed (Lavagna and Pépin, 1999 and 2000) a self-consistent one-loop approximation for the $S=1 / 2$ Kondo lattice model $(N=2)$ that enlarges the standard $1 / N$ expansion theories up on the spinfluctuation effects in complete analogy with the self-consistent renormalized theory of spin fluctuations (SCR-SF) developed for the Hubbard model. It results a quantum-classical crossover at finite temperature depending whether the temperature is lower or higher than the characteristic energy scale of the damped collective mode existing in the vicinity of the magnetic instability. We refer to the paper (Lavagna and Pépin, 2000) for the discussion of the related phase diagram at $d=2$ and $d=3$ with the predictions of a series of crossovers in the vicinity of the quantum critical point as reported in figure 4.

\section{Conclusion}

To conclude, we will say that the pertubative renormalization group (RG) approach and the related self-consistent renormalized spin fluctuation (SCR-SF) theory by eliminating the short-range details of the fluctuations of the order parameter, assume that the low-energy excitations are spatially extended and by essence do not take into account the critical local nature of the modes at the atomic length scale. As we mentioned at the end of section 2, there exist stong indications now based on INS experiments in heavy-fermions, that the magnetic excitations are critically local around the quantum critical point. This feature seems to be a characteristics of most of the strongly-correlated electron systems including high $T_{C}$ superconductors. The existence of critical local modes is related to the formation of local moments in the ordered phase in contrast to the itinerant magnetism picture that is described by pertubative RG and SCR-SF theories. Note that a scaling law in $\omega / T$ of the dynamical spin susceptibility has been obtained theoretically in the case of spin systems as for the random two-dimensional Heisenberg antiferromagnetic model (Sachdev, 1992). There is an urgent need to develop theories to describe this type of local quantum critical point in itinerant systems. In this perspective, we will mention two recent attempts to do that : on the one hand, calculations based on the 
dynamical mean field theory ( $\mathrm{Si}$ et al, 1999) which have recently lead to scaling law in $\omega / T$ for the dynamical spin susceptibility in agreement with experimental observations; on the other hand supersymmetric approaches (Pépin and Lavagna, 1997 and P. Coleman, Pépin, Tsvelik, 2000) based on a mixed fermionic-bosonic representation of the spin.which has the advantage of capturing both the quasiparticle and the local moment features via respectively the fermionic and bosonic degrees of freedom.

Acknowlegements : I am grateful to Zsolt Gulácsi for giving me the opportunity to give these lectures. I would like to thank Nick Bernhoeft, Andrey Chubukov, Piers Coleman, Mucio Continentino, Jacques Flouquet, Gilbert Lonzarich, Catherine Pépin, Stéphane Raymond, Louis-Pierre Regnault, Almut Schröder and Hilbert von Löhneysen for helpful discussions.

* Also Part of the Centre National de la Recherche Scientifique (CNRS) 


\section{References}

D. Bitko, T.F. Rosenbaum and G. Aeppli, Phys. Rev. Lett. 77, 940 (1996)

E. Brézin and J. Zinn-Justin, Nucl. Phys. B 257, 867 (1985); E. Miranda, V. Dobrosavljevic and G. Kotliar, Phys. Rev. Lett. 78, 290 (1997)

P. Coleman, Physica B 259-261, 353 (1999)

P. Coleman, C. Pépin and A.M. Tsvelik, Phys. Rev. B 62, 3852 (2000); P. Coleman, C. Pépin and A.M. Tsvelik, Nucl. Phys. B 586, 641 (2000)

S. Doniach, Physica B 91, 231 (1977)

J.A. Hertz, Phys.Rev. B 14, 1165 (1976)

J.A. Hertz and M.A. Klenin, Phys.Rev. B 10, 1084 (1974)

M. Lavagna and C. Pépin, Phys. Rev. B 62, 6450 (2000); M. Lavagna and C. Pépin, Acta Physica Polonica B 29, 3753 (1998)

N.D. Mathur, F.M. Grosche, S.R. Julian, I.R. Walker, D.M. Freye, R.K.W. Haselwimmer and G.G. Lonzarich, Nature 394, 39 (1998) and references therein

A.J. Millis and P.A. Lee, Phys.Rev. B 35, 3394 (1987); A. Auerbach and K. Levin, Phys.Rev.Lett. 57, 877 (1986)

A.J. Millis, Phys.Rev. B 48, 7183 (1993)

T. Moriya and A. Kawabata, J. Phys. Soc. Jpn 34, 639 (1973); T. Moriya and T. Takimoto, J. Phys. Soc. Jpn 64, $960(1995)$

C. Pépin and M. Lavagna, Phys. Rev. B 59, 2591 (1999)

C. Pépin and M. Lavagna, Z. Phys. B 103, 259 (1997); C. Pépin and M. Lavagna, Phys. Rev. B 59, 12180 (1999)

S. Raymond, L.P. Regnault, S. Kambe, J.M. Mignod, P. Lejay and J. Flouquet, J.Low Temp.Phys. 109, 205 (1997); S. Kambe, S. Raymond, L.P. Regnault, J. Flouquet, P. Lejay and P. Haen, J. Phys.Soc.Jpn 65, 3294 (1996)

S. Sachdev and J. Ye, Phys. Rev. Lett. 69, 2411 (1992)

S. Sachdev, Quantum Phase Transitions ed. by Cambridge University Press, Cambridge (1999)

For reviews see the Proceedings of the ITP Conference in Non-Fermi Liquid Behavior in Metals, Santa Barbara, US, 17-21 June 1996, eds P. Coleman, B. Maple and A.J. Millis, J. Phys.: Condens. Matter 8, no.48 (1996)

A. Schröder, G. Aeppli, E. Bucher, R. Ramazashvili and P. Coleman, Phys. Rev. Lett. 80, 5623 (1998)

A. Schröder, G. Aeppli, R. Coldea, M. Adams, O. Stockert, H. von Löhneysen, E. Bucher, R. Ramazashvili and P. Coleman, Nature 407, 351 (2000)

C.L. Seaman, M.B. Maple, B.W. Lee, S. Ghamaty, M.S. Torikachvili, J.-S. Kang, L.-Z. Liu, J. Allen and D.L. Cox, 
Phys.Rev.Lett. 67, 2882 (1991)

Q. Si, J.L. Smith and K. Ingersent, Int. J. Mod. Phys. B 13, 2331 (1999); J.L. Smith and Q. Si, Europhys. Lett. 45, 228 (1999); Q. Si, S. Rabello, K. Ingersent and J.L. Smith, cond-mat/0011477 (2000)

F. Steglich, B. Buschinger, P. Gegenwart, M. Lohmann, R. Helfrich; C. Langhammer, P. Hellmann, L. Donnevert, S. Thomas, A. Link, C. Geiber, M. Lang, G. Sparn and W. Assmus, J. Phys.: Condens. Matter 8, 9909 (1996)

H. von Löhneysen, A. Schröder, M. Sieck and T. Trappmann, Phys.Rev.Lett. 72, 3262 (1994); H. von Löhneysen, J.Phys.Cond.Matt. 8, 9689 (1996) and references therein 


\section{Figure captions}

Figure 1: Schematic phase diagram as a function of temperature $T$ and dimensionless coupling $g$. The full line $T_{C}$ is the line of phase transitions separating the long-range ordered and disordered phases. The upper dotted line is the Ginzburg temperature. An effective classical theory apply in the region located between the two dotted lines surrounding $T_{C}$. Regime I is the quantum disordered regime characterized by quantum fluctuations. Regime III is the thermally disordered regime in which all the physical quantities are driven by temperature only. Regime II is the intermediate quantum critical regime in which both quantum and classical fluctuations are relevant. The arrows below the graph indicate how $g$ can be tuned by a chemical composition change $x$ or pressure $P$.

Figure 2 : Illustration on the scaling procedure in $q$ and $\omega$. The shaded region represents the outer-shell contribution associated with the sum $\Sigma$ " over the $q$ and $\omega$ variables. The complementary inner-shell contribution corresponds to the sum $\Sigma^{\prime}$ over $q$ and $\omega$.

Figure 3: Continuum of electron hole pair excitations. The dotted line represents the damped collective mode exhibiting a change in the dispersion at a characteristic energy $\omega^{*}$ and wavevector $\xi^{-1} \sim \sqrt{\delta}$. The energyscale $\omega^{*}$ defines the crossover temperature $T_{I}$ separating the quantum from the classical regime.

Figure 4: Phase diagram in the plane $(T, I)$ for dimension $d$ equal to 3 and $z=2$ for the Kondo lattice model (Lavagna and Pépin, 2000). The shaded region represents the long-range antiferromagnetic phase bordered by the Néel temperature $T_{N}$. The unshaded region marks the magnetically-disordered regimes $I, I I$ and $I I I$ associated with different behaviors of the system. Regime $I$ is the quantum regime in which the energy of the relevant mode on the scale of $\xi$ is much greater than $k_{B} T$. Regime $I I$ and $I I I$ are both classical regimes in which the thermal effects are important since the fluctuations on the scale of $\xi$ have energy much smaller than $k_{B} T$. In Regime $I I, \xi$ is still controlled by $(1-I)$ but the staggered spin susceptibility is sensitive to the thermal fluctuations. In Regime $I I I$, both $\xi$ and $\chi_{Q}^{\prime}$ are controlled by the temperature. 


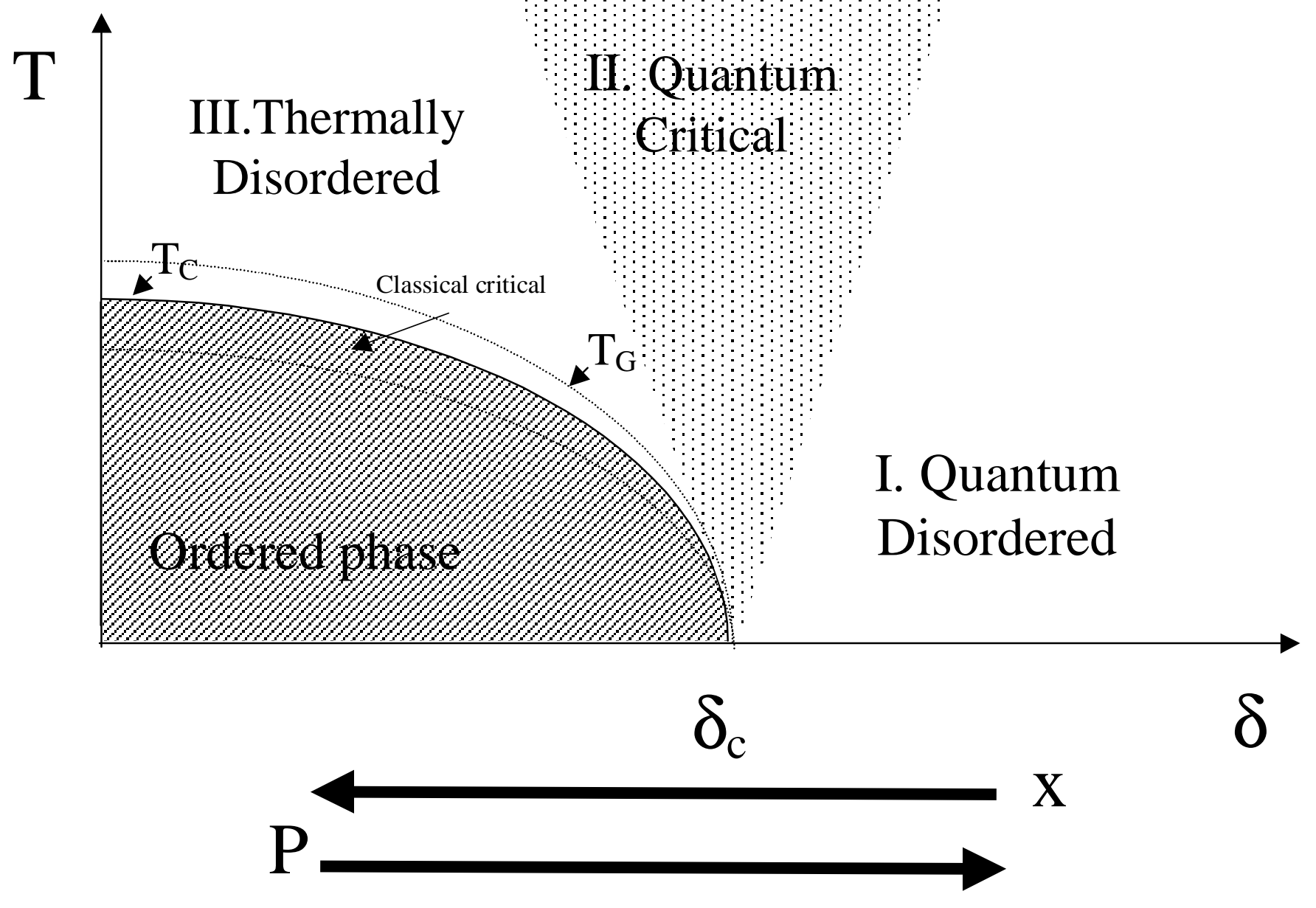




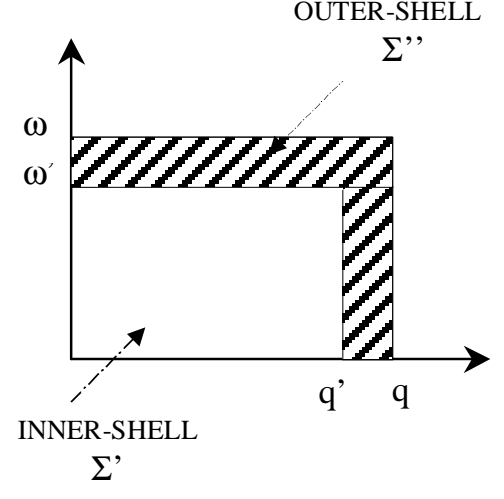




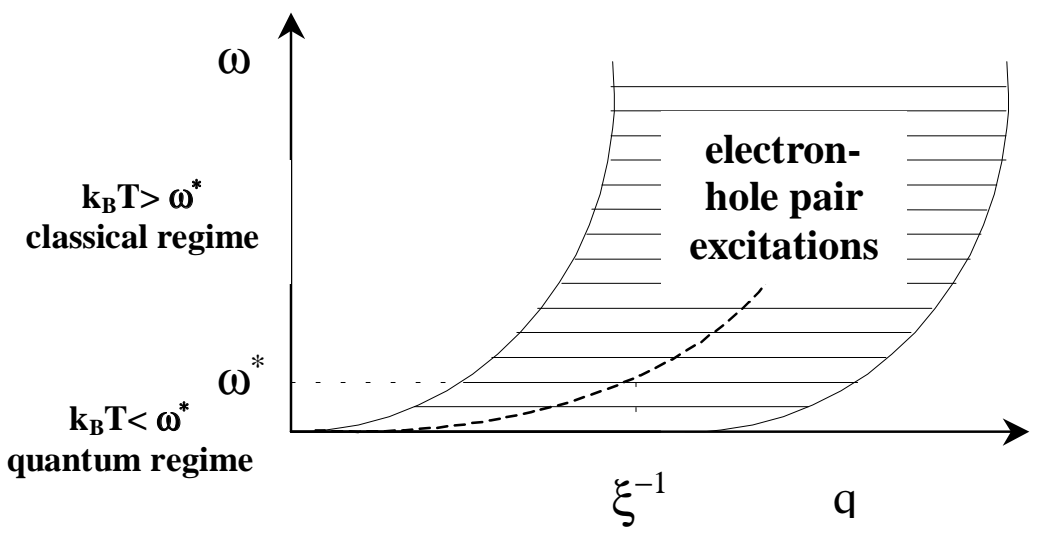




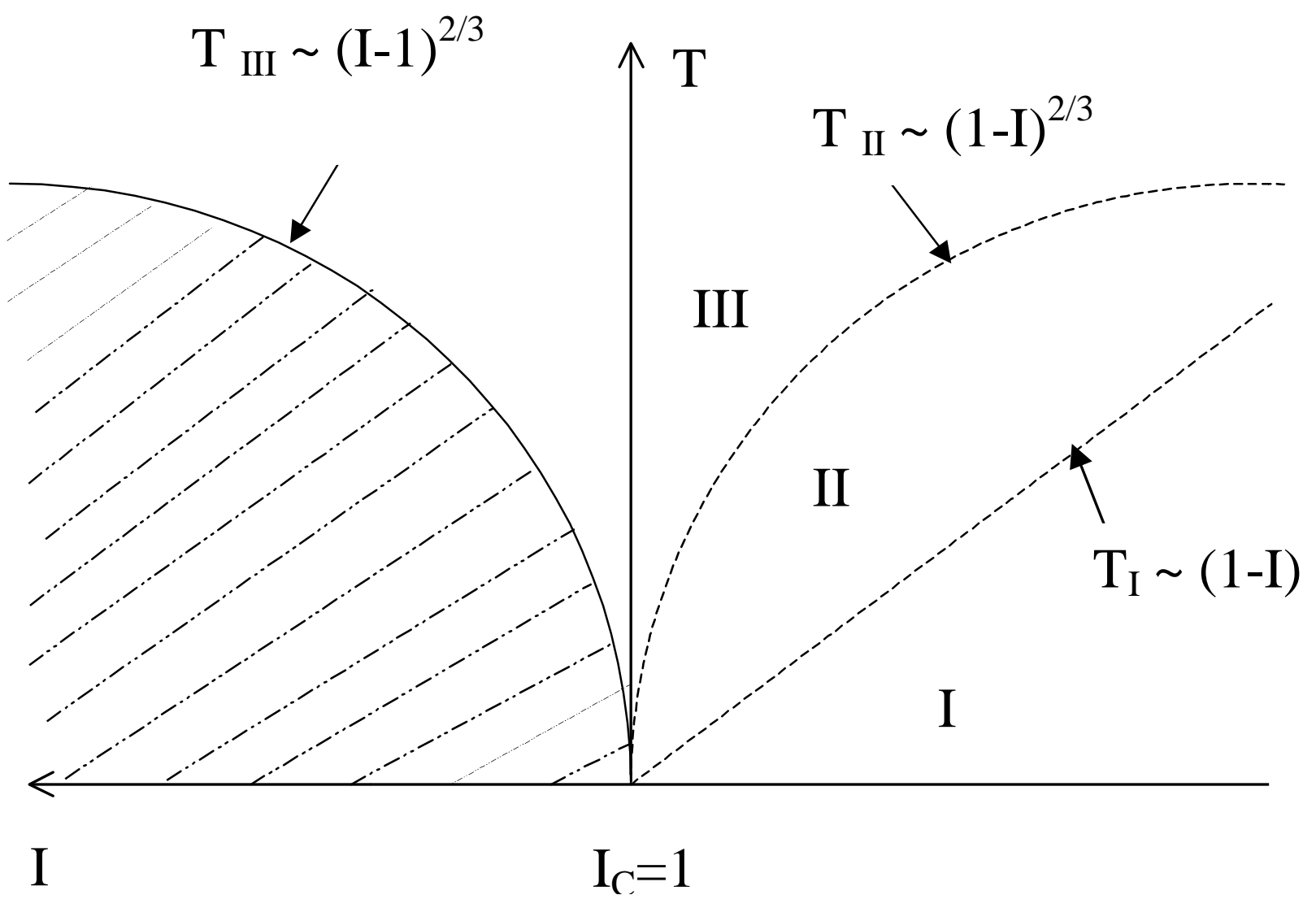

